\title{
A General Lagrangian Approach to Simulate Pollutant Dispersion in Atmosphere for Low-wind Condition
}

\author{
Jonas C. Carvalho',", Marco T. M. B. Vilhena ${ }^{2}$, Gervásio A. Degrazia ${ }^{3}$, Marie li Sallet ${ }^{1}$ \\ ${ }^{1}$ Universidade Federal de Pelotas, Pós-Graduação em Meteorologia, Pelotas, RS, Brazil \\ ${ }^{2}$ Universidade Federal do Rio Grande do Sul, Pós-Graduação em Eng. Mecânica, Porto Alegre, RS, Brazil \\ ${ }^{3}$ Universidade Federal de Santa Maria, Pós-Graduação em Meteorologia, Santa Maria, RS, Brazil
}

\begin{abstract}
In this work we present a semi-analytical Lagrangian particle model to simulate the pollutant dispersion during low wind speed conditions. The model is based on a methodology, which solves the Langevin equation through the assumption that coefficient of the integrating factor is a complex function. The method leads to a non-linear stochastic integral equation, which is solved by the Method of Successive Approximations or Picard's Iterative Method. Taking into account the isomorphis m between the complex and real plane by writing down the low wind formulation in polar form, the procedure allow to determine a formula for the low wind direction. Furthermore, an expression analogous to the Eulerian autocorrelation function suggested by Frenkiel[1] appears in the real component solution. The model results present an improve ment in relation to the other models and are shown to agree very well with the field tracer data collected during stable conditions at Idaho National Engineering Laboratory (INEL).
\end{abstract}

Keywords Low W ind Speed Condition, Air Pollution Modelling, Lagrangian Particle Model, Picard Iterative Method, Autocorrelation Function, Model Evaluation

\section{Introduction}

Recent years have been seen the flowering of the work of searching analytical solution for the Langevin equation with the main purpose of simulating pollutant dispersion in the atmosphere. The mean ing of analytic ity relies on the fact that no approximation is made in the derivatives or domain discretization along the solution derivation. In this direction, appeared in the literature the works of Carvalho et al.[2-3], which solves the Langevin equation by the following steps: linearization of the Langevin equation and solution of the resultant stochastic integral equation by the Picard iterative scheme. This procedure leads to an analytical solution in each iterative step.

Carvalho and Vilhena[4] solved by this methodology the Langevin equation for low wind speed condition. In order to model the pollutant dispersion during meandering effect in the solution, the authors made the assumption that the coefficient of the integrating factor of the first order linear differential equation is a complex function, the imaginary component models the low wind condition. Furthermore, the authors considered only the real component of the integrating factor. At this point, it is relevant to mention that

* Corresponding author:

jonas.carvalho@ufpel.edu.br (Jonas C. Carvalho)

Published online at http://journal.sapub.org/ajee

Copyright (C) 2013 Scientific \& Academic Publishing. All Rights Reserved by this procedure, the Frenkiel[1] autocorrelation function naturally appears in the solution.

In this work we obtain a more general model, unlike the work of Carvalho and Vilhena[4], considering the real and imaginary parts of the complex function before performing the multip lication of the integrating factor, exp ressed by the Euler formula, inside and outside of the integral solution. Taking into account the isomorphism between the complex and real plane by writing down the low wind formulation in polar form, the procedure allow to determine a formula for the low wind direction. Furthermore, an expression analogous to the Eulerian autocorrelation function suggested by Frenkiel[1] appears in the real component solution. Finally, it is necessary to mention that when the non-dimensional quantity that controls the meandering oscillation frequency goes to zero this solution reduces to the solutions encountered by Carvalho et al.[2-3] for windy condition. The low wind speed data collected during stable conditions at Idaho National Engineering Laboratory (INEL)[5] has been used to evaluate the new model. The paper is outlined as follows: in section two the model is presented, in section three the modelling results are discussed and in section four the conclusions.

\section{The Low Wind Model}

The approach consists in the linearization of the Langevin equation as stochastic differential equation: 


$$
\frac{d U}{d t}+f(t) U=g(t)
$$

which has the well known solution in terms of the integrating factor:

$$
U=\frac{1}{\int_{t_{0}}^{t} f\left(t^{\prime}\right) d t^{\prime}} \int_{t_{0}}^{t} g\left(t^{\prime}\right) e^{\int_{t_{0}}^{t} f\left(t^{\prime}\right) d t^{\prime}} d t^{\prime}
$$

In order to embody the low wind speed condition in the Langevin equation, it is assumed that $U$ and $f(t)$ are complex functions written as:

$$
U=u+i v
$$

and

$$
f(t)=p+i q
$$

where $u$ and $v$ are the real and imaginary parts of $U$, respectively, and $p$ and $q$ are the real and imag inary parts of $f(t)$, respectively. Therefore, the exponentials appearing in Equation (2) reads like:

$$
e^{\int_{t_{0}}^{t} f\left(t^{\prime}\right) d t^{\prime}}=e^{p t+i q t} .
$$

Applying the Euler formula, Equation (2) becomes:

$$
\begin{gathered}
U=e^{-p t}[\cos (q t)+i \sin (q t)][u(0)+i v(0)]+ \\
\int_{t_{0}}^{t} g\left(t^{\prime}\right)\left[\frac{e^{-p t}[\cos (q t)-i \sin (q t)] \times}{e^{-p t^{\prime}}\left[\cos \left(q t^{\prime}\right)-i \sin \left(q t^{\prime}\right)\right]}\right] d t^{\prime}
\end{gathered}
$$

Multiplying the Equation (6) by the complex conjugate and performing the multiplications, we can obtain

$$
\begin{gathered}
U=e^{-p t}[\cos (q t)+i \sin (q t)][u(0)+i v(0)]+ \\
\int_{t_{0}}^{t} g\left(t^{\prime}\right) e^{-p\left(t-t^{\prime}\right)}\left\{\cos \left[q\left(t-t^{\prime}\right)\right]-i \sin \left[q\left(t-t^{\prime}\right)\right]\right\} d t^{\prime}
\end{gathered}
$$

Considering $t-t^{\prime}=\tau$, we can write the Equation (7) as

$$
\begin{aligned}
U= & e^{-p t}[\cos (q t)+i \sin (q t)][u(0)+i v(0)]+ \\
& \int_{t_{0}}^{t} g\left(t^{\prime}\right) e^{-p \tau}[\cos (q \tau)-i \sin (q \tau)] d t^{\prime}
\end{aligned}
$$

In order to determine the wind direction we cast equation (8) into:

$$
\begin{aligned}
U= & e^{-p t} \cos (q t) u(0)+\int_{t_{0}}^{t} g\left(t^{\prime}\right) e^{-p \tau}[\cos (q \tau)] d t^{\prime}+ \\
& e^{-p t} \sin (q t) v(0)-\int_{t_{0}}^{t} g\left(t^{\prime}\right) e^{-p \tau}[\sin (q \tau)] d t^{\prime},
\end{aligned}
$$

where by comparison with Equation (3) we have

$$
u=e^{-p t} \cos (q t) u(0)+\int_{t_{0}}^{t} g\left(t^{\prime}\right) e^{-p \tau}[\cos (q \tau)] d t^{\prime}
$$

and

$$
v=e^{-p t} \sin (q t) v(0)-\int_{t_{0}}^{t} g\left(t^{\prime}\right) e^{-p \tau}[\sin (q \tau)] d t^{\prime}
$$

Bearing in mind the isomorphis $m$ between the complex and real planes, the low wind expression given by Equation (9) is described in the complex plane. This procedure allows as determining the low wind direction, using polar form. For this end, we rewrite Equation (9) like

$$
U=\sqrt{u^{2}+v^{2}} e^{i \theta}
$$

where $\theta$ is the low wind direction relative the $x$-axis

$$
\theta=\arctan \left(\frac{v}{u}\right)
$$

Note that the real component of the Equation (9), $e^{-p \tau}[\cos (q \tau)]$ is analogous to the Eulerian autocorrelation function suggested by Fren kiel[1] (p. 80) and written in a different way by Murgatroyd[6]. There fore, $p$ and $q$ are given by

$$
p=\frac{1}{\left(m^{2}+1\right) T} \text { and } q=\frac{m}{\left(m^{2}+1\right) T}
$$

where $T$ is the time scale for a fully developed turbulence and $m$ is a non-dimensional quantity that controls the meandering oscillation frequency. At this point, it is important to mention that when $m$ goes to zero the Equation (9) reduces to the solution for windy conditions, which is written in terms of the exponential form of the autocorrelation function.

The Equation (9) is a non-linear stochastic integral equation, which must be solved iteratively. The method applied to solve the Equation (9) is the Method of Successive Approximations or Picard's Iteration Method[7], assuming that the initial guess for the iterative approximation is determined from a Gaussian distribution. For applications, the values for the parameters $m$ and $T$ have been calculated according to Carvalho et al.[8]:

$$
m=\frac{T_{*}+\sqrt{T_{*}^{2}-16 \pi^{2} T^{2}}}{4 \pi T}
$$

and

$$
T=0.064 \frac{h}{u_{*}},
$$

where $h$ is the stable PBL height, $u_{*}$ is the friction velocity and $T_{*} \cong 2000 \mathrm{~s}$.

As the turbulence is considered Gaussian in the horizontal direction, the function $g\left(t^{\prime}\right)$ can be given by: 


$$
g\left(t^{\prime}\right)=\frac{1}{2} \frac{\partial \sigma_{i}^{2}}{\partial x_{j}}+\frac{u_{i}^{2}}{2 \sigma_{i}^{2}}\left(\frac{\partial \sigma_{i}^{2}}{\partial x_{j}}\right)+\left(\frac{2 \sigma_{i}^{2}}{\tau_{L_{i}}}\right)^{1 / 2} \xi_{i}\left(t^{\prime}\right),
$$

where $\sigma^{2}$ is the turbulent velocity variance, $\tau_{L}$ is the Lagrangian time scale and $\xi_{i}$ is a normally distributed (average 0 and variance $d t$ ) random increment. For the vertical component, we solve the Langevin equation by the ILS approach as suggested by Carvalho et al.[4].

\section{Modelling Results}

The data utilized to evaluate the model performance are composed by a series of field experiments conducted under stable conditions in low winds over flat terrain. The tracer data were collected at Idaho National Engineering Laboratory (INEL) and the results are published in a U.S. National Oceanic and Atmospheric Admin istration (NOAA) report[5].

For the simulations, the turbulent flow is assumed inhomogeneous only in the vertical and the transport is realized by the longitudinal component of the mean wind velocity. The horizontal domain was determined according to sampler distances and the vertical doma in was set equal to the observed PBL height. The time step was maintained constant and it was obtained according to the value of the Lagrangian decorrelation time scale $\left(\Delta t=\tau_{L} / c\right)$, where

$\tau_{L}$ must be the smaller value between $\tau_{L_{i}}$ (with $i=1,2,3$ ) and $c$ is an empirical coefficient set equal to 10 . The values of $\sigma_{i}$ and $\tau_{L_{i}}$ were parameterized according to scheme developed by Degrazia et al.[9]. The integration method used to solve the integrals appearing in Equation (10) was the Romberg technique.

Because of wind direction variability during the INEL experiment, a full $360^{\circ}$ sampling grid was implemented. Arcs were laid out at radii of 100, 200 and $400 \mathrm{~m}$ from the emission point. Samplers were placed at intervals of $6^{\circ}$ on each arc for a total of 180 sampling positions. The receptor height was $0.76 \mathrm{~m}$. The tracer $\mathrm{SF}_{6}$ was released at a height of $1.5 \mathrm{~m}$. The $1 \mathrm{~h}$ average concentrations were determined by means of an electron capture gas chromatography. Wind measurements were provided by lightweight cup anemometers and bivanes at the 2, 4, 8, 16, 32 and $61 \mathrm{~m}$ levels of the $61-\mathrm{m}$ tower located on the $200 \mathrm{~m}$ arc. Table 1 shows the meteorological data utilized for the validation of the proposed model.

Observed wind speeds were used to calculate the coefficients for the exponential wind profiles. The Monin-Obukhov length $L$ and the friction velocity $u_{*}$ were approximated through the numerical best fit between the observed wind speeds and calculated wind profile suggested by Businger et al.[10]. To calculate $h$ (the stable PBL height), the relation $h=0.4\left(u_{*} L / f_{c}\right)^{1 / 2}$ was used[11], where $f_{c}$ is the Coriolis parameter.

The model performance is shown in Tables 1 and 2 and Figures 1. Table 1 shows the comparison between observed and predicted ground-level centerline concentrations. Table 2 presents the result of the statistical analysis made with observed and predicted values of ground-level centerline concentration following Hanna's[12] statistical indices. Giving a look at the results we observe that the model simu lates quite well the experimental data in stable condition and, indeed, presents results comparable or even better than ones obtained by Carvalho and Vilhena[4] and Carvalho et al.[8]. The statistical analys is reveals that all indices are within acceptable ranges, with NMSE, FB and $F S$ values are relatively near to zero and $R$ and $F A 2$ are relatively near to 1 .

Table 1. Observed and predicted ground-level cent erline concentrations

\begin{tabular}{|c|c|c|c|}
\hline run & distance (m) & $\begin{array}{c}\text { observed }(\mu \\
\left.\mathrm{gm}^{-3}\right)\end{array}$ & $\begin{array}{c}\text { predicted }(\mu \\
\left.\mathrm{gm}^{-3}\right)\end{array}$ \\
\hline 4 & 100 & 155 & 160 \\
\hline 4 & 200 & 80 & 70 \\
\hline 4 & 400 & 39 & 29 \\
\hline 5 & 100 & 48 & 41 \\
\hline 5 & 200 & 31 & 22 \\
\hline 5 & 400 & 11 & 11 \\
\hline 7 & 100 & 45 & 47 \\
\hline 7 & 200 & 25 & 30 \\
\hline 7 & 400 & 36 & 17 \\
\hline 8 & 100 & 36 & 34 \\
\hline 8 & 200 & 13 & 29 \\
\hline 8 & 400 & 13 & 8 \\
\hline 9 & 100 & 44 & 44 \\
\hline 9 & 200 & 23 & 22 \\
\hline 9 & 400 & 16 & 12 \\
\hline 10 & 100 & 45 & 47 \\
\hline 10 & 200 & 34 & 17 \\
\hline 10 & 400 & 13 & 2 \\
\hline 11 & 100 & 38 & 40 \\
\hline 11 & 200 & 18 & 15 \\
\hline 11 & 400 & 18 & 2.5 \\
\hline 12 & 100 & 58 & 60 \\
\hline 12 & 200 & 52 & 24 \\
\hline 12 & 400 & 29 & 25 \\
\hline 13 & 100 & 65 & 80 \\
\hline 13 & 200 & 48 & 25 \\
\hline 13 & 400 & 28 & 6 \\
\hline 14 & 100 & 60 & 67 \\
\hline 14 & 200 & 34 & 38 \\
\hline 14 & 400 & 6 & 3 \\
\hline
\end{tabular}

Table 2. Statistical evaluation

\begin{tabular}{|c|c|c|c|c|c|}
\hline Model & NMSE & $R$ & $F A 2$ & $F B$ & $F S$ \\
\hline Proposed Model & 0.10 & 0.94 & 0.80 & 0.12 & -0.11 \\
\hline Carvalho and Vilhena[4] & 0.11 & 0.93 & 0.83 & 0.02 & -0.18 \\
\hline Carvalho et al.[8] & 0.14 & 0.94 & 0.77 & 0.08 & -0.26 \\
\hline
\end{tabular}




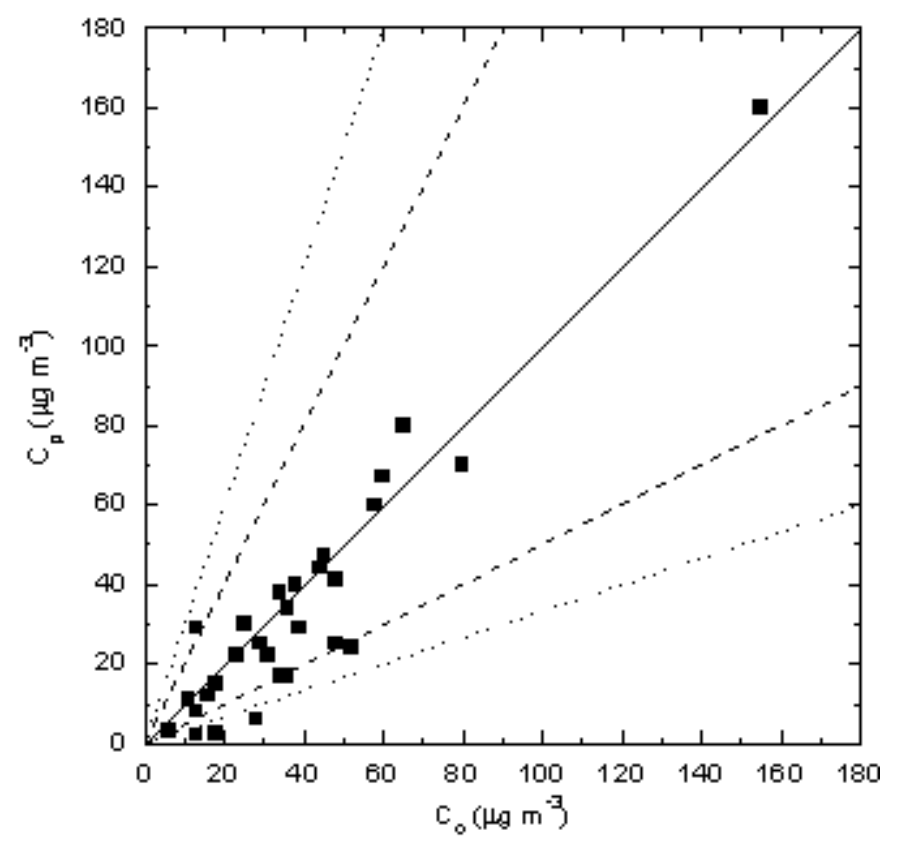

Figure 1. Scatter diagram between observed $\left(C_{0}\right)$ and predicted $\left(C_{p}\right)$ ground-level centerline concentration. Dashed lines indicate fact or of 2 , dotted lines indicated factor of 3 and solid line indicates unbiased prediction

\section{Conclusions}

In this paper was presented a more general model to simulate the pollutant dispersion in meandering low wind conditions. The model was obtained by solving the Langevin equation through the integrating factor with its coefficient being a complex function. The method leads to a stochastic integral equation whose solution was obtained through the Method of Successive Approximations or Picard's Iteration Method. Taking into account the isomorphism between the complex and real plane by writing down the low wind formulation in polar form, it was possible to determine a formula for the low wind direction. An expression analogous to the Eulerian autocorrelation function for meandering conditions appeared in the real component solution. The proposed method can be used to simulate de contaminant dispersion in meandering or non-meandering situations. The model was evaluated through the comparison with experimental data. The results obtained by the new model agree very well with the experimental data, indicating that it represents the dispersion process correctly in low wind speed conditions.

\section{ACKNOWLEDGEMENTS}

The authors acknowledge the financial support provided by CNPq. (Conselho Nacional de Desenvolvimento Científico e Tecnológico) and CAPES (Coordenação de Aperfeiçoamento de Pessoal de Nível Superior).

\section{REFERENCES}

[1] François N. Frenkiel, "Turbulent diffusion: Mean concentration distribution in a flow field of homogeneous turbulence", Advances in Applied Mechanics, vol.3, pp.61-107, 1953.

[2] Jonas C. Carvalho, Ézio R. Nichimura, Marco T. M. B. Vilhena, Davidson M. Moreira, Gervásio A. Degrazia, "An iterative langevin solution for contaminant dispersion simulation using the Gram-Charlier PDF", Environmental Modelling and Software, vol.20, no 3, pp. 285-289, 2005.

[3] Jonas C. Carvalho, Marco T. M. B. Vilhena and Davidson M. Moreira, "An alternative numerical approach to solve the Lan gevin equation applied to air pollution dispersion", Water, Air and Soil Pollution, vol.163, no 1-4, pp. 103-118, 2005.

[4] Jonas C. Carvalho, Marco T. M. B. Vilhena, "Pollutant dispersion simulation for low wind speed condition by the ILS method", Atmospheric Environment, vol.39, pp.6282-6288, 2005.

[5] Jerrold F. Sagendorf, C. Ray Dickson, "Diffusion under low wind-speed, inversion conditions", U.S. National Oceanic and Atmospheric Administration Tech. Memorandum ERL ARL-52, 1974.

[6] R. J. Murgatroyd, Estimations from geostrophic trajectories of horizontal diffusivity in the mid-latitude troposphere and lower stratosphere, Quarterly Journal of the Royal Meteorological Society, vol.95, pp.40-62, 1969.

[7] W. Boyce, R. DiPrima, 2001. Elementary differential equations and boundary value problems, Wiley, New York, $749 \mathrm{pp}, 2001$.

[8] Jonas C. Carvalho, Gervásio A. Degrazia, Sérgio G. Magalhães, "Parameterization of meand ering phenomenon in a stable atmospheric boundary layer", Physica A, vol.368, pp.247-256, 2006.

[9] Gervásio A. Degrazia, Osvaldo L. L. Moraes, Amauri P. Oliveira, "An Analytical method to evaluate mixing length scales for the planetary boundary layer", Journal of Applied 
Meteorology, vol.35, pp.974-977, 1996.

[10] J. A. Businger, Turbulent transfer in the atmospheric surface layer. In D.A. Hau gen (Ed.), Workshop in Micrometeorology, American Meteorological Society, Boston, pp.67-100, 1973

[11] Sergei S. Zilitinkevith, On the determination of the height of the Ekman boundary layer. Boundary-Layer Meteorology vol.3, pp.141-145, 1972.

[12] Steven R. Hanna, "Confidence limit for air quality models as estimated by bootstrap and jacknife resampling methods", Atmospheric Environment, vol.23, pp.1385-1395, 1989. 